\title{
EFECTO DE UN PRODUCTO LÁCTEO CON PROBIÓTICOS Y PREBIÓTICOS SOBRE LA FUNCIÓN DIGESTIVA DE SUJETOS SANOS Y CONSTIPADOS
}

\author{
EFFECT OF A MILK PRODUCT CONTAINING \\ PROBIOTICS AND PREBIOTICS ON THE DIGESTIVE \\ FUNCTION OF HEALTHY AND CONSTIPATED SUBJECTS.
}

\author{
Martin Gotteland, Marcela Vizcarra, Eduard Maury. \\ Laboratorio de Microbiología y Probióticos. \\ Instituto de Nutrición y Tecnología de los Alimentos (INTA), Universidad de Chile. Santiago, Chile.
}

\begin{abstract}
Constipation is a complex disorder which affects an important part of the population and results in an increase of gastrointestinal symptoms and in lower stool frequency. The addition of soluble fibers (prebiotics) and probiotics in foodstuffs has been proposed as a useful tool to improve digestive wellbeing of affected individuals. The aim of this study was to evaluate the effect of the daily consumption of Minishot, a milk drink with prebiotics (inulin I polydextrose) and probiotics (Bifidobacterium lactis BB12), on the digestive comfort of healthy and constipated subjects. We conducted a randomized, controlled, double blind clinical trial in 33 healthy subjects and 64 constipated subjects ( $\leq 3$ stools / week). Each subject underwent a one-week observation period followed by two periods of two weeks of consumption of Minishot or placebo, separated between them by a two-week washout period. The volunteers recorded daily in a table the presence and severity of distractors and digestive symptoms and the number and consistency of stools. Neither Minishot nor the placebo affected the digestive wellbeing or the stool frequency and consistency in the control subjects. In the constipated subjects, both consumption of Minishot and the placebo improved digestive comfort and stool frequency. However, compared with the placebo, Minishot significantly decreased the frequency of hard stools and the proportion of subjects emitting them, and increased the frequency of normal stools.
\end{abstract}

Key words: prebiotics, probiotics, constipation, digestive wellbeing.

Este trabajo fue recibido el 11 de Mayo de 2010 y aceptado para ser publicado el 5 de Julio de 2010.

\section{INTRODUCCIÓN}

La constipación es un trastorno complejo y frecuente del funcionamiento intestinal asociado a diversas patologías y que puede ser un efecto secundario al consumo de fármacos. Es una condición crónica que afecta en general a los individuos por períodos prolongados; en la mayoría de los pacientes se instala progresivamente en ausencia de síntomas que sugieran una enfermedad de base y en los que se descubren factores causales asociados, entre otros, con la dieta y la falta de ejercicio físico (1).

En los países occidentales, los individuos que consumen una dieta normal producen unos $100 \mathrm{~g} / \mathrm{d}$ de depo- siciones, con un contenido de agua cercano al 70-75\%. El número de normal de evacuaciones varía considerablemente de un individuo a otro, y oscila normalmente entre tres y diez por semana, lo que quiere decir que un número inferior a tres evacuaciones semanales definiría a la constipación $(1,2)$. Otros factores ayudan a completar la definición, tales como la necesidad de tener que pujar fuertemente para expulsar las heces, la sensación de que la evacuación ha sido incompleta, la evacuación de bolos duros o fragmentado (escíbalos) después de ingerir laxantes, la sensación de obstrucción anorectal y, por último, el empleo de maniobras manuales para facilitar la defecación completan la definición de constipación; 
esta sintomatología debe estar presente en al menos 25\% de las evacuaciones. En la mayoría de los pacientes que alegan estar crónicamente constipados, la principal queja es la dificultad para evacuar en ausencia de defecaciones infrecuentes (2).

Se estima que cerca del $40 \%$ de las consultas al policlínico de Gastroenterología Pediátrica del Hospital Clínico San Borja-Arriarán, cuya cobertura es de unos 300.000 habitantes se deben a constipación y que la cifra es parecida respecto de los adultos (3). En Estados Unidos se ha calculado que cada año se producen unos dos y medio millones de consultas a especialistas por esta causa y que el gasto en laxantes es de aproximadamente 800 millones de dólares. Hay que tener en cuenta que la mayor parte de los sujetos aquejados de constipación no consulta a su médico (4).

Una manera de disminuir la constipación a través del manejo nutricional del sujeto afectado es a través del consumo de probióticos y de prebióticos. Los probióticos son bacterias vivas, principalmente bifidobacterias y lactobacilos, que son incorporadas a alimentos y que al ser ingeridas modifican actividades fisiológicas del organismo $(5,6)$. Algunos probióticos modulan la actividad motriz y absortiva del colon y podrían modificar su flora residente en los pacientes constipados; lo que podría ser de particular interés pues se ha descrito recientemente alteraciones de la microbiota intestinal en el sujeto constipado, con niveles menores de las bifidobacterias comparado con los sujetos sanos (7).

Por otra parte los prebióticos son polisacáridos no absorbibles que llegan al colon donde son fermentados por la microbiota residente $(5,8)$. Los más consumidos son los fructooligosacáridos, galactooligosacaridos y almidón resistente que son compuestos naturales provenientes de vegetales. También existen prebióticos sintéticos como la polidextrosa, que es ampliamente utilizada por las empresas de alimentos (9).

Entre los efectos de los prebióticos están la estimulación del crecimiento de las bifidobacterias, el aumento del agua y del peso de las deposiciones, el aumento de la producción de ácidos grasos de cadena corta mediante su fermentación por la microbiota, el aumento de la absorción de calcio desde el colon (5). En la actualidad se considera que para que pueda tener efecto prebiótico, un producto con fibra soluble debe contener a lo menos $1.5 \mathrm{~g}$ por porción y que el nivel mínimo de ingestión debería ser $3 \mathrm{~g} / \mathrm{d}$, con un máximo aconsejado de $30 \mathrm{~g} / \mathrm{d}$. En efecto, la única limitación para el uso de estos compuestos en los alimentos es la tolerancia gastrointestinal a su ingestión, ya que el consumo de cantidades elevadas puede producir meteorismo, borborigmo, sensación de distensión abdominal, dolor abdominal y deposiciones blandas que pueden llegar hasta la diarrea Ensayos clínicos indican que una dosis de hasta $30 \mathrm{~g} /$ día de inulina es bien tolerada por sujetos sanos mientras que otros muestran que la ingestión de $12 \mathrm{~g}$ diario de polidextrosa mejora la función intestinal sin ejercer efectos adversos $(10,11)$. Estas fibras solubles, por lo tanto, pueden también contribuir a aliviar la sintomatología de los sujetos constipados $(12,13)$.

En base a estos antecedentes, el objetivo de este estudio fue evaluar el efecto del consumo de un producto lácteo comercial (Minishot) con un probiótico (Bifidobacterium lactis Bb12) y con prebióticos (inulina + polidextrosa) sobre la función digestiva de sujetos asintomáticos y constipados.

\section{SUJETOS Y MÉTODOS Diseño}

Se realizó un ensayo clínico randomizado, controlado y en doble ciego, que incluyó un periodo de observación de una semana seguido por 2 periodos de 14 días de consumo del producto o de su placebo, separados entre si por un periodo de reposo de 14 días. Se eligió un periodo de consumo de los productos de 2 semanas basado en estudios anteriores que indican que el consumo de productos con prebióticos y/o probióticos afecta rápidamente la microbiota intestinal y la función o bienestar digestivo de los consumidores $(14,15)$. Uno de los principales objetivos del periodo de observación, además de entregar valores basales de los parámetros estudiados, fue confirmar que los sujetos constipados realmente lo eran. En efecto, en un estudio anterior realizado también en sujetos constipados, nos dimos cuenta que una proporción importante de ellos sobreestimaban su problema y que en realidad, su estado no respondía a los criterios que definen la constipación (16). Los dos periodos de consumo fueron distribuidos en forma aleatoria en cada sujeto. Cada ensayo se realizó en forma independiente en dos grupos de sujetos, asintomáticos y constipados. Los sujetos debían consumir diariamente una unidad del producto o del su placebo durante cada uno de los periodos de prueba. Se les solicitó a los voluntarios que no consumieran otros yogures, leches cultivadas o productos probióticos durante la duración del estudio. A cada voluntario se le entregó semanalmente una tabla de síntomas incluyendo síntomas confundentes o distractores y síntomas digestivos, donde debía registrar cada día la intensidad (graduada de 0 a 3: nula, leve, moderada, fuerte) percibida para cada uno de ellos. También debía registrar en otra tabla el número de deposiciones emitidas diariamente en función de su consistencia (muy dura, dura, semi-dura, normal, 
blanda, muy blanda o líquida), ayudándose de las ilustraciones presentes en la escala Bristol distribuida junto con la tabla de síntomas (figura 1).

\section{Reclutamiento de los sujetos}

Se reclutaron mujeres mayores de edad quienes padecían de constipación, definida como una frecuencia de evacuación menor de tres veces por semana y con además 2 o más de los siguientes síntomas: necesidad de esfuerzo desmedido para defecar, sensación de eva- cuación incompleta o de obstrucción anorectal, deposiciones duras o en masas duras, necesidad de maniobras manuales para facilitar la defecación.

Se reclutaron también mujeres de características similares, pero que no padecían de constipación. La presencia del estado "constipado" o "no-constipado" fue confirmada por el análisis de la sintomatología y del número de deposición emitida durante la semana de observación previo al principio del estudio.

Se excluyeron de ambos estudios los sujetos con

\section{FIGURA 1}

Escala Bristol de consistencia de las deaposiciones.

\begin{tabular}{|l|l|}
\hline Type I & $\begin{array}{l}\text { Separate hard lumps, like nuts } \\
\text { (hard to pass) }\end{array}$ \\
\hline Type 2 & Sausage-shaped but lumpy \\
\hline itke a sausage but with cracks on & Soft blobs with clear-cut edges \\
\hline and soft & (passed easily) \\
\hline Type 5 & Fluffy pieces with ragged edges, a \\
\hline Type 6 & Wyshy stool \\
\hline Type 7 & Watery, no solid pieces. \\
\hline
\end{tabular}

Tipo 1: Deposición en masas duras y separadas, en forma de nueces (que pasan difícilmente); Tipo 2: Deposición con forma de salchicha, pero con forma grumosa o irregular; Tipo 3: Deposición en forma de salchicha, más lisa pero con grietas en la superficie; Tipo 4: Deposición normal, en forma de salchicha o serpiente, lisa y blanda; Tipo 5: Deposición en masas suaves y pequeñas con bordes claramente definidos; Tipo 6: Deposición en masas esponjosas con bordes difusos, una única evacuación esponjosa; Tipo 7: Deposición acuosa sin masas sólidas, totalmente líquida. 
diabetes o otra enfermedad crónica, con diarrea, con patologías gastrointestinales previas, con un consumo actual o reciente de antibióticos, anti-inflamatorios, laxantes u otras drogas, o con alteración de la anatomía o de la función de la pared muscular del abdomen, todas situaciones que interfieran con la defecación.

\section{Ética}

El protocolo fue aprobado por el Comité de Ética del INTA. A los sujetos se les explicó en forma detallada los objetivos y métodos del estudio; en caso de aceptar de participar en el estudio, debieron firmar un consentimiento informado.

\section{Productos}

Los productos (Minishot y placebo) fueron entregados en forma semanal por la empresa (Soprole, Santiago, Chile) y se almacenaron a $4^{\circ} \mathrm{C}$ en la cámara fría hasta su distribución a los voluntarios. Ambos productos tenían un aspecto idéntico, diferenciándose entre si sólo por la presencia de un código en la tapa. La composición del Minishot esta descrita en la tabla 1. Las concentraciones del probiótico $\left(>10^{7} \mathrm{UFC} / \mathrm{ml}\right)$ en el producto fueron confirmadas a lo largo del estudio por un laboratorio externo. El placebo era el mismo producto pero sin $\mathrm{Bb} 12$ y sin los prebióticos. Al principio de cada semana de los periodos de consumo, se les entregaba a los voluntarios 8 productos $(7+1$ por la dilución familiar) junto con las tablas de sintomatología, las cuales debían ser devueltas completadas al principio de la semana siguiente. El ciego (es decir el código) de los productos fue mantenido hasta haber realizado el estudio estadístico de los datos.

\section{Análisis estadístico}

Para cada síntoma se sumaron las intensidades registradas diariamente durante las dos semanas de cada periodo de consumo. Se promediaron los valores obtenidos para cada síntoma de manera de obtener un grado de intensidad por semana que pueda ser comparado a los valores obtenidos durante la semana de observación, que fue considerada como el periodo basal del estudio. Los resultados fueron expresados como promedio $\pm \mathrm{DE}$ o EEM y/o como mediana y rango interquartil (RIQ). Se compararon los valores de sintomatología obtenidos en el periodo basal de observación entre los dos grupos, control y constipado, utilizando la prueba no-paramétrica de Mann-Whitney. Además de comparar cada uno de los síntomas (distractor o digestivo) por si solo, también se analizaron el total de los distractores y el total de los síntomas digestivos. Los resultados obtenidos durante cada uno de los tres periodos (observación y consumo) para cada síntoma, fueron comparados entre si por análisis de varianzas no-paramétricas de Friedman para muestras pareadas. En caso de ser positivos los resultados por el análisis de varianza, se realizaron las comparaciones entre el producto y su placebo. Estos mismos análisis se realizaron también con el número de deposición emitida en función de su consistencia. Los porcentajes de sujetos emitiendo un tipo de deposición particular fueron comparados por Chi-cuadrado. Estos análisis se realizaron en forma independiente para cada grupo, sano y constipado.

\section{TABLA 1}

\section{Composición nutricional del producto utilizado en el estudio (Minishot).}

\section{Composición nutricional}

Energía (Kcal)

Proteínas $(\mathrm{g})$

Grasa total $(\mathrm{g})$

Colesterol (mg)

Azucares totales (g)

Fibra soluble: inulina + polidextrosa $(\mathrm{g})$

Sodio (mg)

Cepas starters: (L. bulgaricus, S. thermophilus, L. acidophilus)

Cepa probiótica (B. lactis Bb12)

\section{1 envase $=1$ porción}

$(100 \mathrm{ml})$ 


\section{RESULTADOS \\ Caracterización de los grupos}

En el grupo control sano se reclutaron 33 sujetos (edad: $34.8 \pm 13.9$ años; índice de masa corporal (IMC): $23.2 \pm 2.6 \mathrm{~kg} / \mathrm{m} 2)$. De ellos 28 completaron el estudio lo que significa una tasa de abandono del $15.1 \%$, compatible con lo observado en estudios similares. En el grupo constipado 107 sujetos iniciaron el protocolo; sin embargo 43 de estos sujetos tuvieron que ser excluidos después de la semana de observación ya que los resultados mostraron que no respondían a los criterios de constipación previamente definidos. En consecuencia 64 sujetos (edad: $35.7 \pm 15.5$ años; IMC: $24.3 \pm 4.7 \mathrm{~kg} / \mathrm{m} 2$ ) fueron finalmente incluidos en el estudio de los cuales 56 lo completaron, es decir una tasa de abandono de $12.5 \%$.

\section{Periodo inicial de observación}

La intensidad total de los distractores y de la sintomatología digestiva y el número total de deposiciones emitidas durante el periodo de observación en el grupo sano y el grupo constipado aparecen en la figura 2. Los sujetos constipados se diferenciaron fuertemente de los sujetos sanos tanto en la sintomatología digestiva y confundente (distractores), cuyas intensidades son significativamente mayores, como en la emisión de deposiciones que es significativamente menor. Estos resultados confirman que el reclutamiento de los sujetos se hizo en forma adecuada para cada uno de los dos grupos del estudio ya que ambos grupos se diferencian en forma clara. La Tabla 2 muestra en forma individual la intensidad de cada uno de los síntomas distractores y digestivos y el número de deposiciones emitidas según su consistencia durante este mismo periodo inicial. Los sujetos constipados sienten mayor intensidad de casi la totalidad de los síntomas digestivos evaluados y también de muchos $(67 \%)$ de los síntomas confundentes. Tienen una mayor emisión de deposiciones muy duras y una menor emisión de deposiciones más blandas, comparado

\section{FIGURA 2}

\section{Comparación de los distractores, síntomas digestivos y número de deposiciones emitidas entre el grupo control y el grupo constipado durante el periodo de observación.}

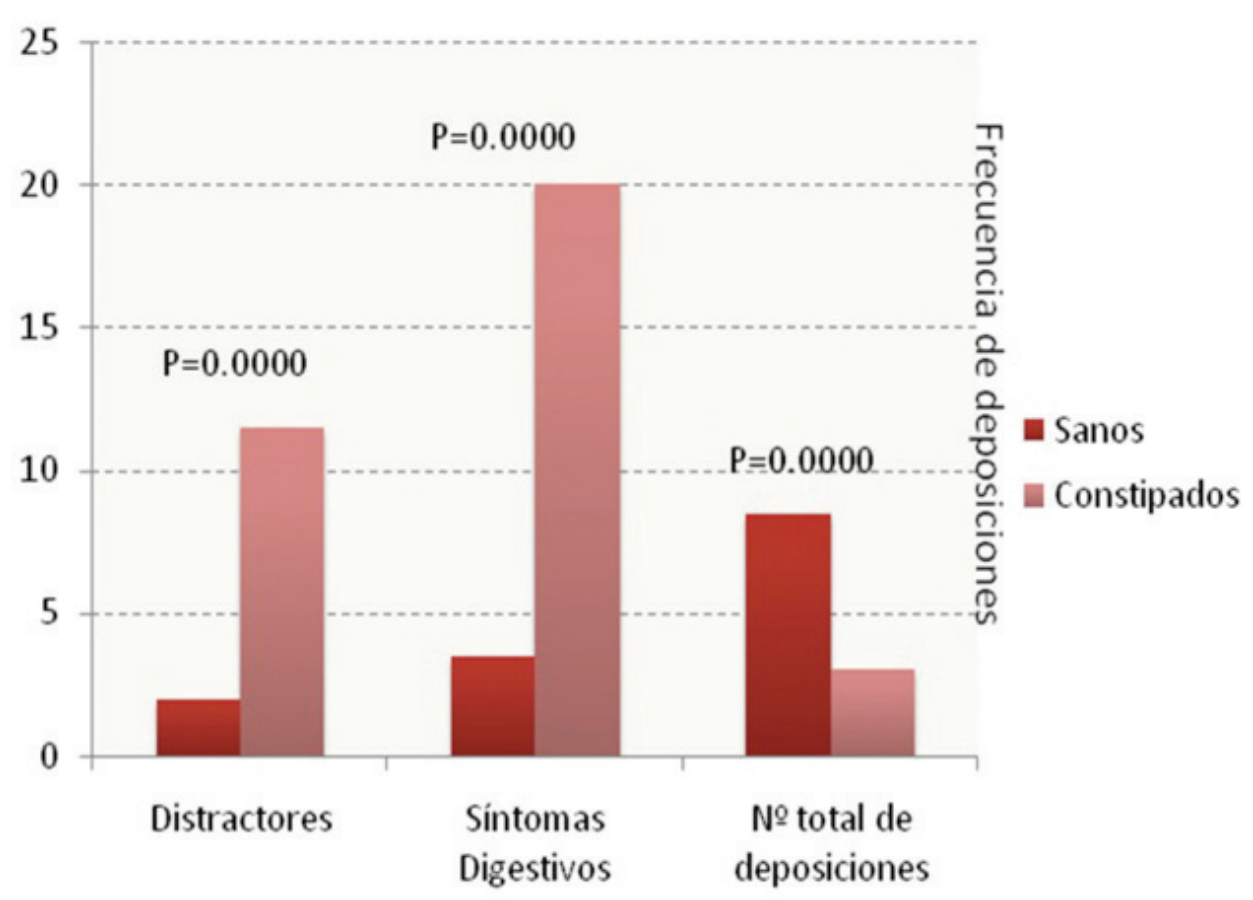

Mediana [rango interquartil]; análisis estadístico por la prueba no-paramétrica de Mann-Whitney.

El eje izquierdo corresponde a la intensidad de los síntomas distractores y digestivos mientras que el eje derecho corresponde a la frecuencia semanal de las deposiciones emitidas (la escala es la misma para ambos ejes). 
con los controles.

\section{Efecto del consumo del Minishot en el grupo control sano}

Se observó una disminución no significativa de la intensidad de los síntomas distractores totales entre el periodo inicial de observación y los periodos de consumo del Minishot y del placebo $(4.57 \pm 1.21 ; 2.43 \pm 0.90 \mathrm{y}$ $2.61 \pm 0.77$, respectivamente; Anova, $\mathrm{p}=0.06$ ) mientras que la sintomatología digestiva total no fue afectada $(5.39 \pm 0.71 ; 4.81 \pm 0.57$ y $5.35 \pm 0.71$, respectivamente). Sin embargo, el análisis individual de los síntomas digestivos mostró una disminución significativa $(\mathrm{p}=0.04)$ del dolor abdominal con el Minishot y con el placebo, comparado con el periodo de observación. En cuanto al número total de deposiciones emitidas por semana, no se observaron diferencias significativas entre el periodo de observación y el periodo de consumo de Minishot o del Placebo $(9,25 \pm 3,92,9,07 \pm 3,61$ y $8,83 \pm 3,89$ deposiciones/semana, respectivamente, $\mathrm{p}>0,05)$. La distribución de las deposiciones emitidas en función de su consistencia esta descrita en la figura 3.

\section{Efecto del consumo del Minishot en el grupo de sujetos constipados}

Como lo muestra la figura $4 \mathrm{~A}$, los resultados muestran un cambio en la intensidad de los síntomas durante el estudio distractores (Anova, $\mathrm{p}=0.034$ ), lo

\section{TABLA 2}

Comparación de los distractores, síntomas digestivos y número de deposiciones emitidas entre el grupo control y el grupo constipado durante el periodo de observación.

\section{Distractores:}

Cefalea

Sequedad de la boca

Prurito cutáneo

Sensación de fatiga

Dolor articular

Dolor de garganta

Visión borrosa

Sensación de fiebre

Insomnio

\section{Síntomas digestivos:}

Dolor abdominal

Vómitos - regurgitaciones

Esfuerzos para defecar

Dolor durante la defecación

Borborismos

Distensión abdominal

Aumento de gases rectales

\section{Deposiciones emitidas:}

Deposición de Tipo 1

Deposición de Tipo 2

Deposición de Tipo 3

Deposición de Tipo 4

Deposición de Tipo 5

Deposición de Tipo 6

Deposición de Tipo 7

$$
\begin{aligned}
& 1.0[0.0-2.5] \\
& 0.0[0.0-1.5] \\
& 0.0[0.0-0.0] \\
& 0.0[0.0-0.0] \\
& 0.0[0.0-0.0] \\
& 0.0[0.0-0.0] \\
& 0.0[0.0-0.0] \\
& 0.0[0.0-0.0] \\
& 0.0[0.0-0.0]
\end{aligned}
$$

$0.0[0.0-1.5]$

$0.0[0.0-0.0]$

$0.0[0.0-0.0]$

$0.0[0.0-3.0]$

$0.0[0.0-2.0]$

$0.0[0.0-0.0]$

$1.0[0.0-3.0]$

$2.0[0.0-4.0]$

$1.0[0.0-2.0]$

$0.0[0.0-1.0]$

$0.0[0.0-0.0]$

\section{CONTROL}

CONSTIPADO

p

$2.0[1.0-4.0]$

0.014

$2.0[0.0-5.0]$

$0.0[0.0-1.0]$

$0.0[0.0-4.0]$

0.0 [0.0 - 1.0]

0.0 [0.0 - 0.0]

0.0 [0.0 - 1.0]

$0.0[0.0-0.0]$

$0.0[0.0-1.5]$

0.001

0.012

0.014

NS

NS

0.038

NS

0.021

$2.0[1.0-7.0]$

0.000

$0.0[0.0-0.0]$

NS

$3.0[2.0-6.0]$

0.000

$2.0[0.0-4.5]$

0.000

$3.0[0.0-7.0]$

0.002

5.0 [0.0 - 9.0]

0.000

$5.0[0.5-8.0]$

0.000

$1.0[0.0-1.5]$

0.004

$0.5[0.0-1.5]$

NS

$0.0[0.0-1.0]$

0.000

$0.0[0.0-0.0]$

0.000

0.0 [0.0-0.0]

0.000

$0.0[0.0-0.0]$

0.000

$0.0[0.0-0.0]$

0.032 


\section{FIGURA 3}

Distribución de las deposiciones emitidas en función de su consistencia durante los tres periodos del estudio en los sujetos sanos.

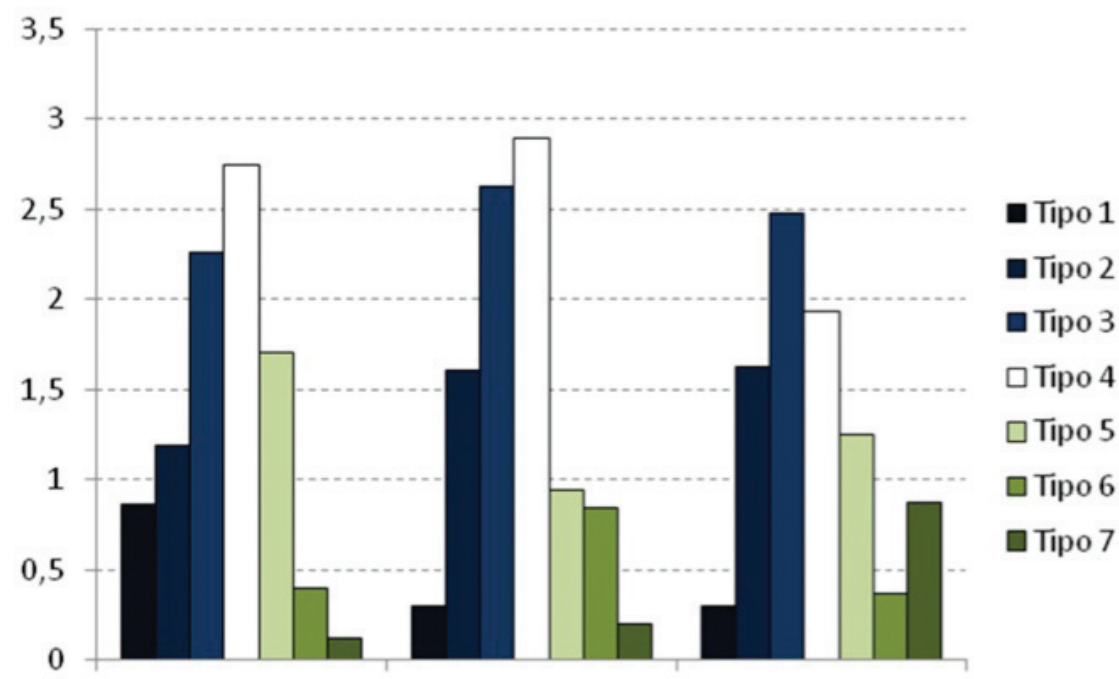

Observación

Minishot

Placebo

\section{FIGURA 4}

Efecto del consumo del Minishot sobre la intensidad de la sintomatología distractora (A) (Anova, $\mathrm{p}=0.034)$ y digestiva total $(B)$ (Anova, $p=0.048$ ) en el grupo constipado.

A

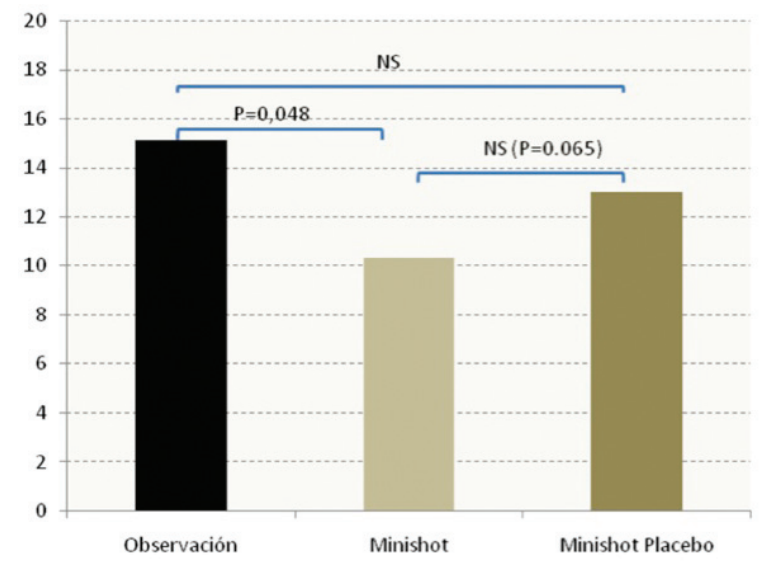

B

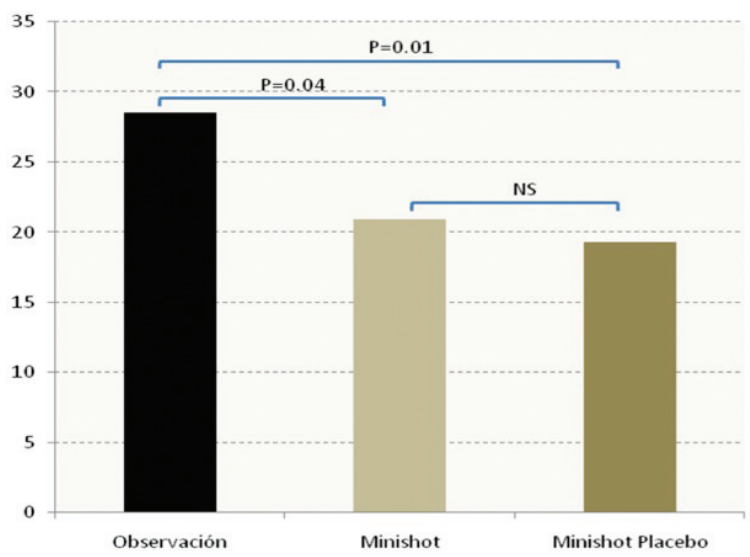


cual refleja una disminución significativa con el Minishot $(\mathrm{p}=0.048)$ pero no con el placebo. El análisis individual de los distractores muestra una disminución significativa ( $\mathrm{p}=0.04)$ del dolor de cabeza con el Minishot (resultados no mostrados). En cuanto a la sintomatología digestiva (figura 4B), no se observaron efectos negativos con el consumo del Minishot o del placebo. Al contrario, la sintomatología digestiva total disminuyó significativamente $(\mathrm{p}=0,048)$ durante el consumo del Minishot pero también del placebo. Tal reducción da cuenta principalmente de la disminución significativa de la intensidad de los síntomas de distensión y dolor abdominal y del esfuerzo al momento de defecar (resultados no mostrados). Sin embargo, estos efectos positivos no pudieron ser atribuidos en forma específica al Minishot. Dichos cambios en la intensidad de la sintomatología digestiva total desaparecieron cuando los resultados fueron normalizados por la intensidad de los distractores, indicando que se podría tratar de un "efecto placebo".

Finalmente, se determinó el efecto del consumo de la administración de Minishot sobre la frecuencia semanal de deposición emitida por los sujetos constipados. La distribución de las deposiciones emitidas en función de su consistencia esta descrita en la figura 5. Tanto el Minishot como el placebo aumentaron el número total de deposiciones emitidas, el cual pasó de $2.59 \pm$ 0.68 deposiciones por semana durante el periodo de observación a $4.98 \pm 1.89$ con el periodo de Minishot y $4.94 \pm 2.35$ con el placebo (Anova, $\mathrm{p}=0.0000$ ), pero $\sin$ alcanzar la frecuencia más alta (7-8/semana) observada en los sujetos sanos. Sin embargo el análisis del perfil muestra diferencias entre los efectos del Minishot y del placebo. En particular, el consumo de Minishot, pero no el de placebo, disminuyó significativamente la emisión de deposiciones muy duras (tipo 1) comparado con el periodo de observación $(\mathrm{p}=0.015)$ (figura 6A). Dicho efecto se observó desde la primera semana de consumo del producto y permaneció durante la segunda semana; además, el porcentaje de mujeres que emitían estas deposiciones muy duras disminuyó significativamente con el Minishot, comparado con el placebo (figura 6B). Por otra parte, tomando en cuenta las deposiciones de tipo 3 a 5 es decir aquellas que pueden se consideradas en el rango de normalidad, se observó que el consumo de Minishot aumento su frecuencia en forma significativa ( $\mathrm{p}=0.0026$ ) y que dicho aumento fue mayor que aquello observado con el placebo $(\mathrm{p}=0,046)$ (figura 5). Este hallazgo esta también soportado por el hecho que el porcentaje de mujeres que emitían estas deposiciones

\section{FIGURA 5}

Distribución de las deposiciones emitidas en función de su consistencia durante los tres periodos del estudio en los sujetos constipados.

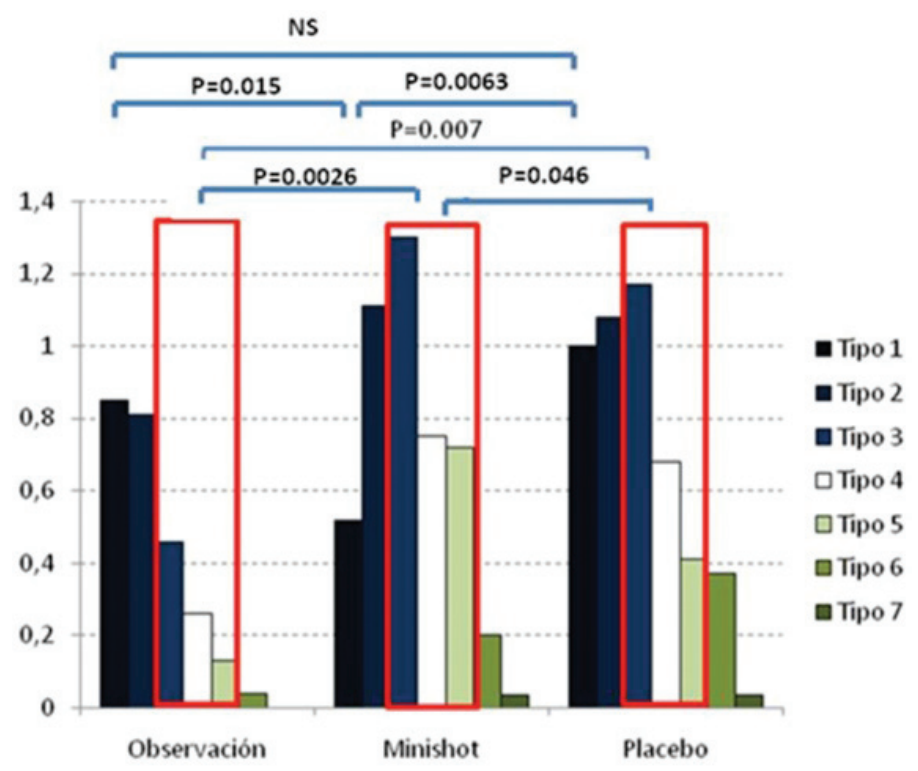


más blandas aumentó significativamente con el Minishot, comparado con su placebo (figura 7).

\section{DISCUSIÓN}

El objetivo del presente estudio fue determinar si el consumo de un producto lácteo con prebióticos (inulina + polidextrosa) y probióticos (B. lactis Bb12), Minishot, podía mejorar el bienestar de sujetos constipados sin afectar negativamente los sujetos sanos sin constipación. En efecto, varios estudios han sugerido la utilización de los prebióticos y probióticos como una herramienta en el manejo nutricional de la constipación $(5,12)$, la cual es considerada como un problema de salud que afecta la calidad de vida de una parte importante de la población y con consecuencias económicas importantes $(1,2)$. Es interesante notar, sin embargo, que parte importante de los sujetos constipados reclutados ( el 40\%), al finalizar el periodo de observación resultaron no ser constipados (según los criterios usados), confirmando que la constipación es frecuentemente sobre-estimada por los individuos (16). En los sujetos reclutados se evaluó por una parte la sintomatología digestiva y por otra parte la frecuencia y consistencia de las deposiciones emitidas. Dado que la evaluación de la intensidad de los síntomas digestivos es muy subjetiva, se determinaron también la intensidad de síntomas distractores (o confundentes), los cuales se eligen por no estar directamente relacionados con los objetivos del estudio y se usan para normalizar los resultados obtenidos con la sintomatología digestiva, disminuyendo de esta forma el componente de subjetividad asociada a esta variable. Cabe destacar que, comparados con los sujetos sanos, los sujetos constipados presentaron no solo una mayor sintomatología digestiva, lo que era esperable, sino también una mayor intensidad de los distractores; esta observación puede ser interpretada como que estos sujetos tienen una sensación de malestar general probablemente asociada con su problema de constipación crónica.

En los sujetos sanos no-constipados, nuestros resultados muestran que el consumo de un Minishot por día no alteró significativamente la intensidad de los distractores y de la sintomatología digestiva, ni la frecuencia y consistencia de las deposiciones emitidas. Se sabe que la tolerancia a los prebióticos incorporados a alimentos es muy variable para una misma dosis, siendo la población clasificada en bajo-, mediano- y alto-respondedores, estos últimos con la capacidad de tolerar dosis de $40 \mathrm{~g}$ sin consecuencias adversas (10-12). La razón de esta variabilidad no esta claramente determinada pero es posible que diferencias cuali o cuantitativas en la microbiota

\section{FIGURA 6}

\section{Porcentaje de las mujeres constipadas que emiten deposiciones muy duras durante los periodos de consumo de Minisho o del placebo.}

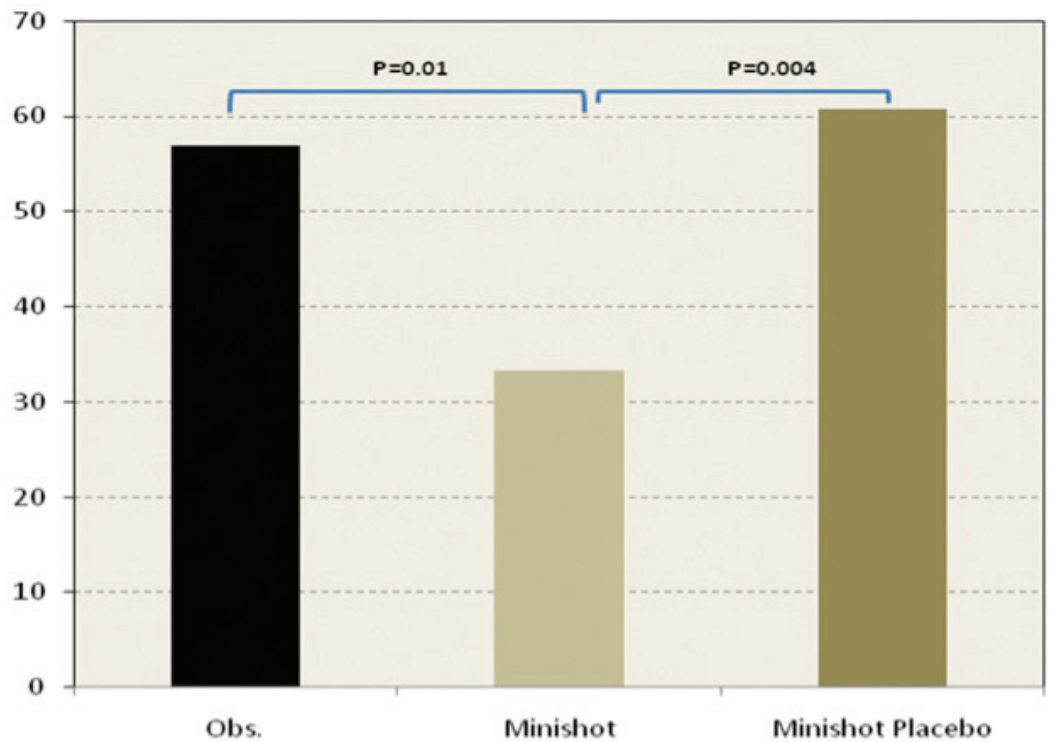


colónica de los sujetos estén involucradas. El producto utilizado en el estudio contenía $3.1 \mathrm{~g}$ de prebiótico, por lo cual aparece como normal que no se haya detectados efectos adversos en los participantes.

En los sujetos constipados, nuestros resultados muestran que el consumo del Minishot o del placebo disminuyó significativamente la sintomatología digestiva. Este resultado es difícil de interpretar debido al hecho que los síntomas distractores también disminuyeron significativamente con ambos productos. En efecto, esta disminución de la sintomatología digestiva esta abolida cuando dichos resultados son normalizados por la intensidad de los distractores, sugiriendo que el consumo de los productos en realidad no afecta la sintomatología digestiva. No obstante, cabe destacar que la intensidad de los distractores normalmente no deberían variar durante los periodos de consumo pues son inespecíficos y no relacionados directamente con el estudio. El hecho que la intensidad de los distractores sea mayor en los sujetos constipados que en los sanos, y que disminuya durante el consumo de los productos confirma que en este estudio, los distractores funcionan más bien como marcadores de la sensación de malestar de los sujetos constipados y que probablemente no pueden ser utilizados para normalizar la sintomatología digestiva.
Por otra parte observamos que el consumo tanto del Minishot como del placebo aumentó significativamente la frecuencia de deposición en las mujeres constipadas, comparado con el periodo inicial. Una posible explicación para la ausencia de diferencia entre Minishot y placebo es la presencia en ambos productos de lactosa (3-4 g) proveniente de la leche. Tomando en cuenta que la mayoría de los participantes deberían ser hipolactásica, la lactosa no digerida podría actuar como fibra en ellos, al igual que la inulina o la polidextrosa (17). Observaciones similares han sido realizadas en otros estudios (14). A pesar de estos resultados, es importante notar que el perfil de deposición emitidas por las mujeres constipadas varia cualitativamente con el consumo de Minishot comparado con el del placebo. En efecto el Minishot, en forma específica, redujo la frecuencia de deposiciones muy duras lo que es un factor importante en determinar el mejoramiento del bienestar digestivo de estos sujetos. De acuerdo con esta observación, la proporción de mujeres que emitían deposiciones muy duras fue significativamente menor que con el placebo. Observamos también que con el Minishot, la emisión de deposiciones normales/blandas aumentó significativamente, a diferencia de lo observado con el placebo; además, la proporción de mujeres que emitían este tipo

\section{FIGURA 7}

\section{Porcentaje de mujeres constipadas con deposiciones blandas (Tipo 3, 4 y 5) durante los periodos de consumo de Minishot o del placebo.}

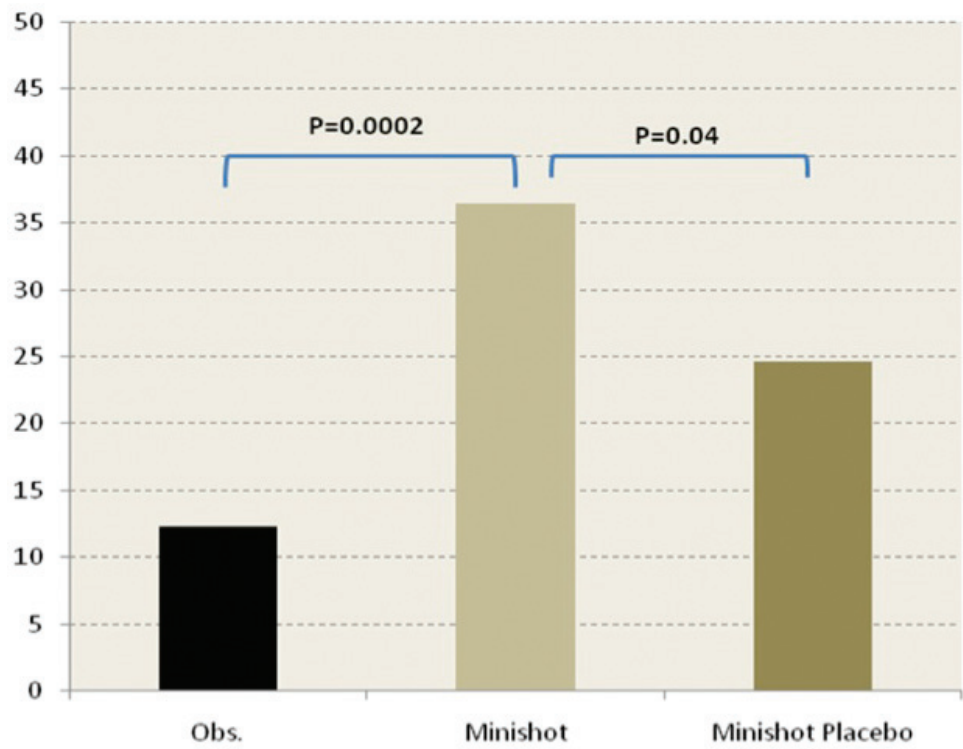


de deposición fue significativamente mayor que con el placebo. Los mecanismos por los cuales los probióticos podrían ejercer estos efectos saludables aún no esta claro. Se ha mostrado que algunas cepas pueden regular el tiempo de tránsito (18). Estos resultados confirman estudios realizados con la misma cepa probiótica $\mathrm{Bb} 12$ (14) o con otras cepas de Bifidobacterium spp o de Lactobacillus y que muestran un mejoramiento del bienestar digestivo y de la frecuencia y/o de la consistencia de las deposiciones en adultos o en niños constipados ( 7 , 15, 19-21).

En conclusión, el consumo de un Minishot por día no afecta el bienestar digestivo de los sujetos sanos ni altera la frecuencia o la consistencia de sus deposiciones. En los sujetos constipados, el consumo de Minishot mejora tanto su bienestar digestivo como la consistencia y frecuencia de las deposiciones. Si bien algunos de estos efectos se observan también por el placebo, el Minishot destaca por su capacidad de disminuir la frecuencia de deposiciones muy duras y la proporción de sujetos que las emiten, y por otra parte en aumentar la frecuencia de deposiciones con consistencia más normal y la proporción de los sujetos que las emiten.

\section{RESUMEN}

La constipación es un trastorno complejo que afecta una parte importante de la población. El consumo de prebióticos y probióticos ha sido propuesta como una herramienta para mejorar el bienestar digestivo de las personas afectadas. El objetivo del estudio fue evaluar el efecto del consumo diario de una bebida láctea con inulina/polidextrosa y Bifidobacterium lactis Bb12, Minishot, sobre el bienestar digestivo de sujetos sanos o constipados. Se realizó un ensayo clínico randomizado, controlado y en doble ciego en 33 sujetos sanos y 64 sujetos constipados ( $\leq 3$ deposiciones / semana). Después de una semana de observación, cada sujeto tuvo 2 periodos de 2 semanas de consumo de Minishot o del placebo, espaciados entre si por 2 semanas de reposo. Los voluntarios registraron diariamente la presencia e intensidad de síntomas digestivos y el número y consistencia de deposiciones. El consumo de Minishot no afecto en forma importante el bienestar digestivo de los sujetos sanos ni alteró la frecuencia y consistencia de sus deposiciones. En los sujetos constipados, tanto el consumo de Minishot como del placebo mejoraron el bienestar digestivo y la frecuencia de deposición. Comparado con el placebo, el consumo de Minishot disminuyó significativamente la frecuencia de deposiciones muy duras y la proporción de sujetos emitiéndolas, y aumento la frecuencia de deposiciones normales/blandas y la proporción de sujetos emitiéndolas.
Palabras clave: prebióticos, probióticos, constipación, bienestar digestivo.

Dirigir la correspondencia a:

Dr. Martin Gotteland

Laboratorio de Microbiología y Probióticos

INTA, Universidad de Chile

El Líbano 5524, Macul

Santiago, Chile

Teléfono: 56-2-9781468

Fax: 56-2-2214030

E-mail:mgottela@inta.c

Agradecimientos: el proyecto fue financiado por Soprole (Chile). Los autores agradecen a los voluntarios por su participación en el estudio.

El auspiciador no tuvo un rol en el diseño del estudio, recolección de la información, análisis de los resultados, interpretación de la información o escritura del artículo. El autor del artículo tuvo acceso completo a toda la información del estudio y la responsabilidad final del envió a publicación del articulo.

\section{BIBLIOGRAFÍA \\ 1. Bharucha AE. Constipation Best Pract Res Clin Gastroenterol 2007; 21: 709-731.}

2. Chatoor D, Emmnauel A. Constipation and evacuation disorders. Best Pract Res Clin Gastroenterol 2009; 23: 517-30.

3. Cofré L P, Germain P F, Medina L L, Orellana G H, Suárez M J, Vergara AT. [Constipation in adults: an update]. Rev Med Chil 2008; 136: 507-16.

4. Corazziari E. Definition and epidemiology of functional gastrointestinal disorders. Best Pract Res Clin Gastroenterol 2004; 18: 613-31.

5. Brunser O, Gotteland M. Prebiotics and probiotics in human health: an overview. In "Bioactive foods in health promotion: probiotics and prebiotic." Watson $\mathrm{R}$ and Preedy V, Ed. Academic press, Kidlingto, UK, 2010.

6. Caceres P, Gotteland M. Alimentos probióticos en Chile: ¿Qué cepas y que propiedades saludables? Rev Chil Nutr. 2010; 37: 97-109.

7. Chmielewska A, Szajewska H. Systematic review of randomised controlled trials: Probiotics for functional constipation. World J Gastroenterol 2010; 16: 69-75.

8. Roberfroid MB. Introducing inulin-type fructans.

9.

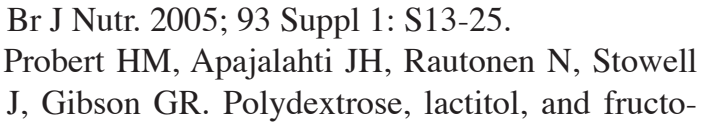


oligosaccharide fermentation by colonic bacteria in a three-stage centinuous culture system. Appl Environ Microbiol 2004; 70: 4505-11.

10. Flood MT, Auerbach MH, Craig SA. A review of the clinical toleration studies of polydextrose in food. Food Chem Toxicol 2004; 42: 1531-42.

11. Hata H, Nakajima K. The relationship between fructo-oligosaccharides intake and intestinal symptoms: observations on the maximum non-effective dose and 50\% effective dose. Geriatr Med. 1985, 23: 817-28.

12. Den Hond E, Geypens B, Ghoos Y. Effect of high performance chicory inulin on constipation. Nutr Res. 2000; 20: 731-736.

13. Kleessen B, Sykura B, Zunft HJ, Blaut M. Effects of inulin and lactose on fecal microflora, microbial activity, and bowel habit in elderly constipated persons. Am J Clin Nutr. 1997, 65:1397-402.

14. Satoshi N, Masami G, Satomi A, et al. Effect of yogurt containing Bifidobacterium lactis BB-12 on improvement of defecation and fecal microflora of healthy female adults Milk Sci 2004. 53. 71-80

15. Yang YX He M Hu G, Wei J, Pages P, Yang XH. Bourdu-Naturel S. Effect of a fermented milk containing Bifidobacterium lactis DN-173010 on Chinese constipated women. World J Gastroenterol 2008; 14: 6237-43.
16. Gotteland M, Brunser O. Efecto de un yogur con inulina sobre la función intestinal de sujetos sanos o constipados. Rev Chil Nutr. 2006; 33: 553-60.

17. Szilagyi A. Lactose - a potential prebiotic. Aliment Pharmacol Ther 2002; 16.1591-602.

18. Marteau F, Cuillerier E, Meance S, et al. Bifidobacterium animalis strain $\mathrm{DN}-173010$ shortens the colonic transit time in healthy women a dowble-hlind, randemized, controlled study. Aliment Pharmacol Ther 2002; 16: 587-93.

19. Simrén M, Ohman L, Olsson J, Svensson U, Ohlson K, Posserud I, Strid H. The effects of a fermented milk containing three probiotic bacteria in patients with irritable bowel syndrome - a randomized, double-blind, controlled study. Aliment Pharmacol Ther $2010 \cdot 31 \cdot 218-27$

20. Guyonnet D, Schlumberger A, Mhamdi L, Jakob S, Chassany O.Fermented milk containing Bifidobacterium lactis DN-173 010 improves gastrointestinal well-being and digestive symptoms in women reporting minor digestive symptoms: a randomised, double-blind, parallel, controlled study. Br J Nutn. $2000 \cdot 102 \cdot 1654-62$

21. De Paula JA, Carmuega E, Weill R Effect of the ingestion of a symbiotic yogurt on the bowel habits f women with functional constipation. Acta Gasroenterol Latinoam 2008; 38: 16-25 